\title{
"A New Physical Aspect for Elastic-Viscous Transition and Velocity Jump in Fracture of Rubbers"
}

\author{
Fukahori Y, Sakulkaew K. and Busfield J.J.C. \\ School of Engineering and Materials science, Queen Mary, University of London \\ Mile End Road, London, E1 4NS, UK
}

\begin{abstract}
The elastic-viscous transition of tear fracture of rubber consists of three different fracture zones, the elastic-brittle fracture zone, the viscous-ductile fracture zone and the intermediate transition zone accompanied with stick-slip motion and a corresponding velocity jump at a constant strain energy release rate. Following the previous paper where the fundamental concept named the elastic-viscous transition for the velocity jump was newly introduced, here in addition the authors propose another new physical concept for the mechanism of the elastic-viscous transition in comparison with the phase transition of water. The vibrational energy caused by the stick-slip motion converted from the external strain energy works as a driving force for the velocity jump, being similar to the situation when the latent heat converted from an external heating source causes a volume jump in the phase transition of water.

The greatly increased vibrational energy might be consumed to accelerate the ductile fracture of the newly induced fraction of the glassy state in the transition process from the rubbery to glassy state around the glass transition region, resulting in the velocity jump at the elastic-viscous transition. It is the purpose of this paper to answer the essential question why the abrupt and significant velocity change occurs under circumstances where the input strain energy release rate is kept constant.
\end{abstract}

\section{KEYWORDS}

Elastic-viscous transition; Strain energy release rate; Stick-slip motion; 


\section{INTRODUCTION}

The energy necessary to drive a crack, is known as the strain energy release rate $\mathrm{G}$ which when based on the Griffith concept [1] is defined as

$$
\mathrm{G}=-\left(\frac{\partial W}{\partial A}\right)_{l}
$$

where $W$ is the total stored elastic energy of the system and $A$ the area of one fracture surface of the crack and $I$ the length of specimen. Fracture occurs when G achieves a critical value $G_{c}\left(G \geq G_{c}\right)$, where $G_{c}$ is equivalent to the intrinsic thermodynamic surface energy for perfectly elastic solid. Rivlin and Thomas [2] and Thomas [3] expanded the Griffith criterion into visco-elastic rubbers, thus the critical value $G_{c}$ measured for the visco-elastic rubbers named tearing energy or fracture toughness is much greater than the thermodynamic surface energy, because its magnitude in addition includes all the energy dissipation in visco-elastic materials during fracture. In practice, fracture results when the external promoting factor for fracture, expressed as the strain energy release rate exceeds the intrinsic resistance to crack growth resulting from energy dissipation in the specimen.

Andrews [4] made the physical meaning of $\mathrm{G}_{\mathrm{c}}$ for visco-elastic polymers much clearer using a function of energy loss as

$$
\mathrm{G}_{\mathrm{c}}=\Phi \mathrm{G}_{0}
$$

where $\Phi$ named a loss function depends on the temperature T, the crack growth rate $\mathrm{R}$ and the strain amplitude $\varepsilon . G_{0}$ is a minimum value of $G_{c}$ for a cross-linked rubber of negligible energy dissipation, equivalent to a case when $\Phi=1$. Thus $\Phi=\Phi(\mathrm{T}, \mathrm{R}, \varepsilon)$ depends strongly on the energy loss of the material characterized by the external conditions of T, $R$ and $\varepsilon$, its value varying from 1 to the level of $10^{2} \sim 10^{3}$. Thus we can restate again the competition between the crack promoting factor $\mathrm{G}$ and the crack resisting factor $\Phi$ determines if crack propagation will take place. The dominant factor also decides the fracture mode and the crack growth rate and this is discussed in detail in this paper.

Greensmith [5] and later Kadir and Thomas [6], Tsunoda et al. [7] and Sakulkaew et al.[8] found important phenomena in tear fracture of rubber in the relation between strain energy release rate $G$ and rate of tear $R$. When the rate of tear approaches the velocity of elastic wave in the rubber, strain energy release rate does not increase monotonically with an increase in the rate of tear. The tear behavior of cross-linked rubber falls into three regions as shown schematically in Fig.1. At low rates the tearing is fairly steady but the fracture surface is very rough (zone I ). At high rates the tearing is quite steady and shows the very smooth fracture surface (zoneIII) similar to that of 
glassy thermoplastic polymers. An intermediate zone II exists between the zone I and III, where the tear proceeds in a stick-slip manner and strain energy release rate is almost constant. They also showed that the behavior in zone III is closely associated with the glass transition temperature of the material. The magnitude of strain energy release rate required to generate the intermediate zone II increases when the viscosity or energy dissipation increases.

Recently, Fukahori, Sakulkaew and Busfield [9] performed more experiments in detail and generalized for the first time these phenomena described all above as an elastic-viscous transition phenomenon. The transition thus characterized depends strongly on the balance of the elasticity given by cross-links and the energy dissipation resulting from the viscosity of polymer. A new elastic-viscous transition diagram is introduced to elucidate the mechanism of this phenomenon, where the diagram consists of three zones, each with different fracture modes. These are an elastic-brittle fracture zone I , a viscous-ductile fracture zone III and an intermediate transition zone II between the elastic and viscous zones where the both fracture modes of brittle and ductile coexist together.

In the intermediate transition zone II , strain energy release rate is kept almost constant, nevertheless the crack growth rate increases abruptly and significantly, which can be seen as a velocity jump during the tear fracture of rubber. The transition zone characterized by stick-slip motion is caused in mechanics by the unstable fluctuations of crack growth rate due to the fluctuation in the modulus and energy dissipation of the material around the glass transition region. They proposed, as a basic concept, a mechanism for the transition to be generated at the crack front both theoretically and experimentally with a consideration of the surface roughness formation and generation of stick-slip motion in frictional sliding of the rubber at the crack front.

A similar phenomenon has been observed during the peeling of an adhesive tape [10, 11, 12], where three modes of peeling are generated with different regimes of peeling velocity. When an applied load to pull the tape reaches a critical peeling force, the peeling velocity suddenly becomes significantly higher as the velocity jump and an unstable peeling with stick-slip motion occurs. This phenomenon has been investigated mainly from the viewpoints of dynamics consideration, where is shown a considerable similarity between the tear fracture of rubber and the peeling of adhesive tape. The purpose of this paper is to add more experiments and discussion and as consequence to derive the essential mechanism of the elastic-viscous transition together with the velocity jump from physical understanding point of view, which may also help explain similar fracture responses such as that observed during peeling of adhesive tape. 


\section{EXPERIMENTS}

Since the detail of the experiments was given in the previous paper [9], only the outline is described here. Four species of compound for cross-linked SBR and NR were used, carbon black-filled (50 phr) and unfilled (0) with low cross-link density (S1) and high cross-link density (S3). Both legs of the trouser test piece were clamped in grips and torn in opposing directions. Two test machines were mainly utilized for experiments depending on the pulling velocity, an INSTRON type machine at the lower velocities and a drop-weight apparatus at the high velocities.

A critical value of the stress required to initiate the crack propagation was read from the point of the first reduction of force in the force-time curve. This value was converted into a critical strain energy release rate at the crack tip $G_{\text {tip }}$. Hence, the time derivative of $\mathrm{G}, \mathrm{dG} / \mathrm{dt}(=\dot{\mathrm{G}})$ just before the critical tearing is given by the slope of the $\mathrm{G}$ - time curve, where $\dot{G}$ is also easily converted into the crack growth rate at the crack tip, $R_{\text {tip }}$, because the strain energy release rate $G$ is given as a function of the crack length $c$, and thus $\mathrm{dG} / \mathrm{dt}$ is combined directly with the crack growth rate $\mathrm{dc} / \mathrm{dt}$.

\section{RESULTS}

\subsection{Relation between the critical strain energy release and the crack growth rate at the tip of a crack}

Fig. 2 shows the Gtip - $\dot{G}$ curves rearranged from the previous results [9] based on a new standpoint for the four different cross-linked SBR compounds, which includes an unfilled highly cross-linked compound (SBR0-S3) and an unfilled less highly cross-linked compound (SBR0-S1) as well as a carbon black-filled highly cross-linked compound (SBR50-S3) and another less cross-linked filled compound (SBR50-S1). It is clearly shown that the tear fracture behavior for all the compounds falls into three zones in the Gtip against $\dot{G}$ relation where the boundary between zone I and zone II is divided by a dashed line (A). In Fig.2. the critical strain energy release rate $G_{t i p}$ increases as a power function of the time derivative $\dot{G}$ in zone I and also in zone III with different slopes. Thus with a quasi-constant slope $n$ for the zone I and III in the log Gtip - logG curve, we can write as

$$
\mathrm{G}_{\text {tip }}=\mathrm{k} \dot{\mathrm{G}}^{\mathrm{n}}
$$

The value of $n$ is roughly estimated as $1 / 7\left(=n_{I}\right)$ for the zone I and $5 / 4\left(=n_{\text {III }}\right)$ for the zone III, indicating that the value of $n_{I}$ is almost $1 / 9$ of that of $n_{\text {III }}$ and both the values seem to be independent of the compound compositions.

Between these two zones, a flat zone (Zone II) appears on the $G_{\text {tip }}-\dot{G}$ curves, where 
the critical strain energy release is almost constant, independent of the crack growth rate. More exactly, the $\mathrm{G}_{\text {tip }}$ seems not to be constant in zone II but changes slightly as shown with a curved dotted line drawn only for the case of SBR50-S1. This is similar to the supersaturated state given in the van der Waal's plot. At the present stage, however, we do not refer to this subtle any further and treat the zone II as a Gtip flat region. In Fig2, it is apparent that the materials of higher visco-elastic energy dissipation such as the lower cross-link density and those filled with carbon black cause a significant increase in $\mathrm{G}_{\text {tip }}$ value over all crack growth rates. In such materials, the time derivative range, interpreted as the width of the intermediate zone II , also becomes wider as energy dissipation increases.

Fig.3 shows the behavior of four different cross-linked NR compounds rearranged from the previous results. For all the NR compounds almost the same phenomena as shown for the SBR compounds (Fig.2) are observed in zone ( I ), ( II ) and (III). Between zone ( I ) and (II), however, appears a new zone (IV) between the two dashed lines (A) and (B). This region appears to indicate a considerable increase in the critical strain energy release rate caused by strain-induced crystallization. Crystallization, of course, requires enough time for molecules to orientate and crystallize and thus the effect of crystallization appears more readily at the lower test rates than are present in zone (II) and consequently the zone (II) expands wider towards lower rates on the rate axis. This graph also highlights how the crystallization can contribute to resisting fracture processes.

\subsection{Characteristics of stick-slip motion generated in the transition zone II}

Fig.4 shows typical profiles of the force-time curve measured in the three zones for the unfilled NR (Fig.4a, NR0-S1 and NR0-S3) and unfilled SBR (Fig.4b, SBR0-S1 and SBR0S3). In the elastic zone I, although the force is not a constant with a similar jagged pattern, its frequency is in the range of $10^{-3} \mathrm{~Hz}$ and thus the force change is not easily measured as a vibration under these general experimental conditions. In the zoneIII, the force is smooth without fluctuations as generally observed in the fracture of glassy polymers (including suitable thermo-plastics). Although the force-time curves observed in the intermediate zone II are somewhat irregular, they indicate a typical jagged pattern known as stick-slip motion with a frequency in the range of $4 \sim 6 \mathrm{~Hz}$.

The most important data in this paper concerning the relation between the frequency of vibration and the crack growth rate measured over very wide ranges of the time derivative $\dot{G}$ for four unfilled compounds is shown in Fig.5, where the frequency is estimated directly from the force-time curve. Three regions are distinguished clearly in 
the log frequency $f$ - $\log \dot{\mathrm{G}}$ curves corresponding to the three different zones I, II and III. In the zone I, the frequency slightly increases from a few $10^{-3}$ to $1 \times 10^{-1} \mathrm{~Hz}$ with increasing $\dot{\mathrm{G}}$ of the range of $10^{-4} \sim \mathrm{a}$ few $10^{-1} \mathrm{kJm}^{-2} \mathrm{~s}^{-1}$ and the frequency is constant in the zoneIII. In the intermediate transition zone II, a linear relation between log $f$ and $\log \dot{G}$ is observed over the frequency ranges from $10^{-1}$ to $3 \times 10^{2}$ corresponding to the $\dot{\mathrm{G}}$ ranges of a few $10^{-1} \sim 1 \times 10^{3} \mathrm{kJm}^{-2} \mathrm{~s}^{-1}$. It is also observed (but not shown here) that the amplitude of the vibration in the force is kept almost constant over the entire transition zone II .

Thus from a straight line with a slope 1 on the plot of $\log f$ - $\log \dot{\mathrm{G}}$ in the intermediate zone II, we can get the proportional relation between the frequency $f$ and $\dot{G}$ for the stick-slip motion in the transition region II as,

$$
f=\mathrm{k} \dot{\mathrm{G}}
$$

where $\mathrm{k}$ is a coefficient. The fact that the frequency increases proportionally to the crack growth rate in the transition region allows us to draw a fundamental and rational basis to understand the physical aspects of the elastic-viscous transition, when considering as to how the stick-slip motion causes the velocity jump in the transition zone II, discussed in detail in the Discussion section.

\subsection{Surface roughness and stick-slip motion in the zone II}

As discussed in detail in the previous paper [9], of three fracture zones the fracture surface shows the highest roughness in the elastic zone I and the lowest in the viscous zone III for all the compounds. The surface roughness of the intermediate transition zone II is also intermediate between the zone I and the zoneIII, and more exactly it can be interpreted as exhibiting simply the mixed feature of the fracture surfaces of zones I and III.

Fig.6 shows SEM photographs for the fracture surface of the intermediate zone II for unfilled compounds NR0-S1, SBR0-S1 and SBR0-S3. It is of great importance to point out that any specific fracture patterns corresponding to the periodic stick-slip motion are not left at all on the fracture surfaces of the intermediate zone II. If fracture occurs in a jagged stick-slip manner, the evidence to show the repetition of different fracture modes or different fracture rates would appear on the fracture surface, as it is the case with the striations perpendicular to the crack propagation observed in the dynamic fatigue fracture process of rubbers. Thus, it is emphasized here that the jagged stick-slip behavior in the force-time diagram does not reflect the direct fracture mechanisms. In other words, the force changes in the force-time plot (Fig.4a and Fig.4b) are not generated during the rupture but after the rapture, independent of the fracture itself. 
In the previous paper [9], we proposed a new approach to help explain this paradox issue. When a primary crack progresses in a rubber, the secondary cracks grown up within the high stress field in front of the primary crack join up with each other and eventually encounter the primary crack, resulting in the crack propagation and the surface roughness formation [13] as shown in Fig.7. During this process, the torn and separated second cracks (two tangs) shrink in an opposite direction to each other due to the entropic elastic retraction force. At that instance, the sliding of the tangs compressed at the crack front with a strong frictional force under a shear stress field might be generated with the stick-slip motion depending on such circumstances as velocity, elasticity and viscosity of the material.

It is well known that stick slip motion is almost always accompanied with the frictional sliding of rubber due to the sticky characters of the rubber surface [14] [15]. It is also verified experimentally that the stick-slip motion occurs around the peak plateau region in the $\tan \delta \sim$ velocity relationship $[16,17,18]$, whereas the peak of the $\tan \delta$ curve can be broadened by suitable compounding for example by filling the compound with carbon black [19]. According to Moore [20], the frictional force depends strongly on the adhesion component within the interaction between solid and rubber, proportional to the energy dissipation factor $\tan \delta$ of the material. Therefore it is quite reasonable to consider that the stick-slip motion is generated accompanied with a frictional sliding of the tangs induced after the rupture of rubber, and as a result a wide flat transition zone (II) results for materials of high energy dissipation with a broad $\tan \delta$ peak, such as carbon blackfilled rubbers as shown in Fig.2 and Fig.3.

\section{DISCUSSION}

\subsection{Elastic-viscous transition diagram for fracture of cross-linked and visco- elastic rubber}

We now, based on all the above data and information, propose a novel universal concept of an elastic-viscous transition in tear fracture of cross-linked and visco-elastic rubbers. The elastic-viscous transition diagram represented newly as a function of $\Phi$ for crosslinked and visco-elastic rubbers in Fig. 8 consists of three discrete fracture mode zones, where $\dot{G}$ is converted to the crack growth rate $R_{\text {tip }}$ at the crack front. At low rates, fracture propagates in a steady and brittle manner (elastic-brittle fracture zone I) and at high rates fracture also propagates in a steady but ductile manner (viscous-ductile fracture zone III). Between the zone I and the zoneIII, the fracture mode changes from the steady to the unsteady response (transition zone II), where the fracture proceeds with a stick-slip manner and the average $G_{\text {tip }}$ value read off on the jagged response is virtually 
constant.

This transition phenomenon is undoubtedly associated with the glass transition behavior of cross-linked rubbery materials. The influence of the energy dissipation for the strain energy release rate works equally over the three zones, resulting in a vertical parallel shifting of the average $\mathrm{G}_{\text {tip }}$ value, that is, the higher the energy dissipation, the higher the critical strain energy release rate. In addition, the width of the transition zone on the crack growth rate axis is also determined by the energy dissipation, a higher energy dissipation generating a wider transition zone. As consequently, the elasticviscous transition diagram has a characteristic shape like the letter $\mathrm{V}$ for the transition zone.

In Fig.8, the energy dissipation is revealed with the loss function $\Phi$ given by equation 2 . When $\Phi$ has a minimum value $(\Phi=1)$, fracture mode changes from the brittle to the ductile directly through the discontinuous change at the glass transition velocity $\mathrm{V}_{\mathrm{g}}$ (corresponding to the glass transition temperature $\mathrm{T}_{\mathrm{g}}$ ). For the case of a perfectly elastic material (non-polymeric solids), the crack growth rate must be fast even at a very low strain energy release rate without any change in the fracture mode, as shown with the linear dotted line in Fig.8. When the magnitude of $\Phi$ is larger than unity $(\Phi>1)$, the transition region appears between the elastic and viscous zones and the higher the magnitude of $\Phi$, then the greater the value of the corresponding $G_{\text {tip }}$ together with the greater width of the transition zone.

Fundamentally, this diagram is closely related to the glass transition behavior of the visco-elastic cross-linked rubber. As shown schematically in Fig.9, in the glass transition region of cross-linked rubber, the modulus change indicated by $\alpha$ is $3 \sim 4$ orders of magnitude in general, whereas the width of the transition zone $\beta$ and hence the width of $\tan \delta$ increase with an increase in the energy dissipation of the compound. Therefore the width of the transition region II in the elastic-viscous transition diagram (Fig.8) seems to be proportional to the width of $\beta$ and $\tan \delta$ in Fig.9. The case of $\Phi=1$ in Fig.8, of course, corresponds to the material with the minimum width $\beta$.

Here we first introduce a new concept for the modulus increase from the rubbery to the glassy state in the glass transition region. In general, although this modulus increase is understood as a gradual increase of the mean modulus of a whole material, but it is also possible to treat the change as the gradual increase of the fraction of the hard glassy state within the mixed rubbery and glassy states. That is, in a system consisting of the both soft rubber and hard glass existing independently, the fraction of the hard glass increases gradually with increasing velocity in the process from rubbery to glassy states in Fig.9. If the volume fraction of the glassy state with ductile fracture is set as $\phi \mathrm{g}, \phi \mathrm{g}$ 
$=0$ corresponds to point $\mathrm{A}$ and $\phi \mathrm{g}=1$ to point $\mathrm{B}$ in Fig.9.

We secondly define the physical concept of the brittle and the ductile fractures using the ratio of the crack growth rate against the strain energy release rate, $\log R_{\text {tip }} / \log \mathrm{G}_{\text {tip. }}$. This definition indicates the effect of the input strain energy release rate for the crack propagation, that is, how easily a crack propagates at lower input energy. From equation 3 with setting $k=1$, the magnitude of $\log R_{\text {tip }} / \log G_{\text {tip }}$, the inverse relation of equation 3 , is given as $1 / \mathrm{n}_{\text {I }}$ for the zone I and $1 / \mathrm{n}_{\text {III }}$ for the zone III and consequently the rate of crack propagation is about 9 times larger for the brittle fracture than for the ductile fracture. In other words, the input strain energy release rate to need to generate the fracture propagation is roughly 9 times larger for the ductile fracture than for the brittle fracture. This difference in fracture modes may result mainly from the difference of molecular packing and viscosity between the rubbery and the glassy states, which, in other words, indicates that the glassy state has a much higher resistance for fracture compared with the rubbery state for cross-linked and visco-elastic polymers.

\subsection{Similarity between the elastic-viscous transition and phase transition of water}

We now take up the phase transition of water $\left(\mathrm{H}_{2} \mathrm{O}\right)$ to consider the physical meaning of the elastic-viscous transition, because of the similarity between both the phenomena. Phase transition of water (volume transition) depends upon the balance of the external condition of temperature and pressure as shown in Fig.10. At a lower temperature and lower pressure than the critical temperature $\mathrm{T}_{c}$ and pressure $\mathrm{P}_{c}$, two phases of water and vapour coexist together. In the transition process from water to vapour the volume increases abruptly whilst both the temperature and the pressure remain constant known as the volume jump.

Under the coexistence condition of water and vapour, thermal energy is always supplied from the outside to the system, despite that the temperature of the system is kept constant. In this process, the excess energy thus imposed is consumed to change the water of low energy state to the vapour of much higher energy state. This excess energy named latent heat is used always to vapourize the water, which is thus the actual driving force to generate the volume jump in the water-vapour transition. That is, the substantial energy imposed by temperature accelerates the volume expansion as a driving force and the surface tension of water behaves against the expansion as a resisting force.

Now we compare the velocity jump in the elastic-viscous transition with the volume jump in the water-liquid transition. The critical condition in the phase transition of 
water (Fig.10), of course, corresponds to the case of $\Phi=1$ in Fig.8, At lower conditions in magnitude than these critical values, the differences between the brittle and the ductile fracture disappears. When $\mathrm{G}_{\text {tip }}$ and $\Phi$ exceed the critical condition, there appears the transition zone where both the brittle and ductile fracture modes coexist. In the transition zone, the strain energy release rate works as a driving force for fracture and the energy dissipation $\Phi$ as a resisting force against fracture, quite similar to the role of the temperature and the surface tension in the water-vapor transition.

As discussed before, much larger strain energy is necessary for the ductile fracture propagation of the hard glassy state compared with the brittle fracture propagation of the soft rubbery state. Therefore, it may be true that during the transition process from the brittle to the ductile fracture response, the additional strain energy is absolutely necessary to be imposed from the outside experimental stress field, because the excess strain energy thus imposed is consumed to drive the ductile fracture of the increased fraction of hard glassy state in the coexisting system according to the modulus increase around the glass transition region. As a consequence, the strain energy release rate is, in appearance, kept constant in the transition zone. This excess strain energy of course plays a role as the latent heat in the phase transition of water.

\subsection{Physical aspects for the elastic-viscous transition and the velocity jump}

Another important question to be answered under the above situation may be as to how the excess strain energy works, as a role of the latent heat, to promote the velocity jump in the elastic-viscous transition. For this question, we must remember the characteristics of the stick-slip motion in the transition zone II shown in Fig.4 and Fig.5. The frequency of stick-slip motion increases by about 3.5 orders of magnitude proportionally to the same increased level of crack growth rate $\dot{G}$ (and thus $\mathrm{R}_{\text {tip }}$ ) of 3.5 orders of magnitude during the transition. The vibrational energy is proportional to the square of frequency of the vibration and in addition, the frequency is proportional to the square root of the stiffness $\mathrm{K}$ (and therefore the modulus of the material E), resulting in the vibration energy $\mathrm{W}_{f}$ being proportional to the modulus $\mathrm{E}$.

Fig.9 highlights that the modulus increases $3 \sim 4$ orders of magnitude in the glass transition region corresponding to the increase of the fraction of the hard glassy state exhibiting a ductile fracture mode. Thus it may be quite reasonable to say that in the intermediate transition zone, the increased vibrational energy $\mathrm{W}_{f}$ being three to four orders of magnitude higher is generated proportionally to the increase of the crack growth rate and it is consumed to drive the ductile fracture of the newly induced fraction of the glassy state into the glass transition region, which of course yields a very rapid 
increase of crack growth rate, and as a consequence creates the velocity jump in the transition zone under the constant strain energy release rate.

\subsection{Role of the vibrational energy to accelerate the crack propagation}

The final question to be answered, therefore, may be how the vibration energy, in a practical sense, does accelerate the crack propagation in tear fracture of rubber. For this question, it may be suggestive to recall two methods that are used to promote fracture, firstly when a vibration is imposed during the cutting of metals and secondly during cavitation in a fluid. In cutting and processing of hard metals, the impulse excited by ultrasonic vibration expands the crack tip and accelerates the crack propagation. Although the high frequency is necessary for cutting of the materials of high stiffness and for fast cutting, it is on the order of $10 \mathrm{KHz}$ for cutting of plastics and FRP [21], [22].

Another method is to add the vibration to metal working oils to induce cavitation inside the oil. When the vibration is applied to the working oil, high pressures and low pressures appear in specific locations, resulting in repeated creation and annihilation of cavities [23], [24]. Cavities thus created at low pressure burst at high pressure resulting in the strong impulse waves of higher energy, which generates the fracture of the work substrate or removes substances as is the case when adopting an ultrasonic washing machine.

Now we consider the possible mechanism of the velocity jump induced by stick-slip vibration in tear fracture of rubber. The frequency of stick-slip vibration in the transition zone is on the order of $10^{-1} \sim$ a few hundred $\mathrm{Hz}$, much lower than $10 \mathrm{KHz}$. However, the vibrational energy at these frequency levels may be enough for the acceleration of fracture propagation of very soft rubber and in particular for the slow crack growth rate in the transition zone II. Moreover, it is well established that a vibration of much higher frequency of a few $\mathrm{KHz}$ is always generated coupled with stick-slip vibration in the frictional sliding of rubber. According to Fukahori and Yamazaki [15], [16], when a rigid slider moves over a rubber surface two kinds of vibration are generated, one is stick-slip motion (a) and another is micro-vibrations (b) as shown in Fig.11, where regions I and II correspond to the stick and slip stage, respectively. The micro-vibration with the natural resonance frequency of rubber in the range of a few $\mathrm{kHz}$ is always generated during whole the stick-slip movement.

Thus, it might be reasonable to conclude that the stick-slip vibration together with the micro-vibration may expand the crack tip and initiate cavitation in the visco-elastic soft rubber ahead of the crack tip as shown in Fig.7. Since the vibrational energy increases 
proportionally to the increase of the crack growth rate in the transition zone, the greatly increased energy is consumed to accelerate the ductile fracture of the newly induced fraction of the glassy state dramatically in the glass transition region. This significant energy increase over the transition zone II connects the elastic-brittle fracture zone I to the viscous-ductile fracture zone III, which seems to be a fundamental mechanism of the velocity jump in the elastic-viscous transition of tear fracture of rubber.

We suppose that the above mechanism for the transition phenomenon and the velocity jump in the tear fracture of rubber may work universally to the similar tear fracture behaviors of other visco-elastic polymers, where a large notch exists with a very large stress concentration and a wide range of secondary cracks surround the notch front as discussed in Fig.7. The tearing and the peeling of the specimen are the cases which satisfy these conditions. Two factors occupy the phenomena, one is the external entropic force and the other the visco-elastic energy dissipation, where the dominant component decides the fracture mode and the rate of fracture and hence the behavior is observed as a function of velocity. Thus it may reasonably be assumed that the same mechanism works in the peeling of adhesive tape [10] [12], because quite similar fracture behaviors have been observed there.

\section{Conclusion}

The elastic-viscous transition and the velocity jump in tear fracture of rubber have been discussed from a physics point of view in comparison with the phase transition of water, resulting in the following new conclusions.

(1) The essential point of this paper is to understand the role of the stick-slip motion and the vibrational energy induced through the stick-slip oscillation. The frequency of the stick-slip motion increases proportionally to the increase of the crack growth rate over the transition zone and consequently the vibrational energy also increases proportionally to the square of the frequency.

(2) The greatly increased vibrational energy with increasing crack growth rate may be used to accelerate the ductile fracture of the newly induced increased volume fraction of the glassy state. The vibrational energy converted from the strain energy works as the driving force to accelerate the crack propagation and results in the velocity jump in the elastic-viscous transition of tear fracture of rubber.

(3) The stick-slip vibration together with micro-vibration of a much higher frequency may expand the crack tip and initiate cavitation in the visco-elastic soft rubber and thus accelerate the crack propagation significantly over the transition zone.

(4) These phenomena are quite similar the situation that the latent heat converted 
from an outside heating source causes the volume jump in the phase transition of water.

\section{REFERENCES}

1) Griffith A.A. Phil. Tran. Roy. Soc 1921; A221, 163

2) Rivlin R.S. Thomas A.G. J. Polym. Sci. 1953; 10, 291

3) Thomas A.G. J. Alppl. Polym. Sci. 1960; 3, 168

4) Andrews E.H. J. Mater. Sci. 1974; 9, 887

5) Greensmith H.W. J. Polym. Sci. 1956; 21, 175

6) Kadir A. Thomas A.G. Rubber Chem. Tech. 1981; 54, 15

7) Tsunoda K. Busfield J.J.C. Davies C.K.L. Thomas A.G. J. Mater. Sci. 2000; 35, 5187

8) Sakulaew K. Thomas A.G. Busfield J.J.C. Polym. Test. 2010; 30, 163

9) Fukahori Y. Sakulaew K. Busfield J.J.C. Polymer 2013; 54, 1905

10) Barquins M. Ciccotti M. Int. J. Adhesion and Adhesive 1997; 17, 65

11) Aubrey D.W. Sherriff M. J. Polym. Sci. Polym. Chem. Ed. 1980; 18, 2597

12) Maugis D. J. Mater. Sci. 1985; 20, 3041

13) Fukahori Y. Andrews E.H. J. Mater. Sci. 1978; 13, 777

14) Fukahori Y. Yamazaki Wear 1994; 171, 195

15) Fukahori Y. Yamazaki Wear 1994; 178, 109

16) Fukahori Y. Gabriel P. Busfield J.J.C. Wear 2010; 269, 854

17) Fukahori Y. Liang H. Busfield J.J.C. Wear 2008; 265, 387

18) Wu-Bavouzet F. Clain-Burckbucher J. Buguin A. De Gennes P.G. Brochard-Wyart F. J. Adhesion 2007; 83, 761

19) Payne A.R. "Use of Rubber in Engineering" (1966), Ed. by Allen P.W. Lindley P.B. Payne A.R., Maclaren and Sons, London, p25

20) Moore D.F. "The Friction and Lubrication of Elastomers" (1972), Pergamon, Oxford

21) Kubo S. et al. Adv. Materials Res. 2010; 126, 493

22) Igarashi K. Sato M. Kakinuma Y. Adv. Materials Res. 2011; 325, 482

23) Kodama T. Tomita Y. et al. J. Biomechanical Sci. Eng. 2009; 14, 124

24) Ohoba R. Nippon Kikaigakai-Shi B 1997; 63, 2264

\section{CAPTIONS}

Fig.1: Strain energy release rate against rate relation (schematic) 
Fig.2: Gtip against $\dot{G}$ relations for four SBR compounds

Fig.3: Gtip against $\dot{G}$ relations for four NR compounds

Fig.4a: Typical profiles of the force-time curves of unfilled NR0-S1 and NR0-S3 in the three zones

Fig.4b: As Fig.4a but of unfilled SBR0-S1 and SBR0-S3

Fig.5 Frequency of vibration $\mathrm{f}$ against $\dot{\mathrm{G}}$ relation for SBR (SBR0-S1 and SBR0-S3) and NR (NR0-S1and NR0-S3) over the three zones

Fig.6 Fracture surfaces for NR0-S1, SBR0-S1 and SBR0-S3 in the transition zone, an arrow indicating the direction of crack propagation

Fig.7: Formation of tangs after secondary fracture (schematic)

Fig.8: Elastic-viscous transition diagram to show the critical strain energy release rate $\mathrm{G}_{\text {tip }}$ against crack growth rate $\mathrm{R}_{\text {tip }}$ relation as a function of $\Phi$ in tear fracture of cross-linked visco-elastic rubbers (schematic)

Fig.9: Glass transition behaviors as a function of velocity for cross-linked visco-elastic rubbers (schematic)

Fig.10: Phase transition phenomena of water (schematic)

Fig.11: Typical spectra of stick-slip motion (frictional force against time, upper) together with micro-vibration (acceleration against time, lower) generated on the surface of rubber when a blade slider slides on rubber block [14] 


\section{Figures}

Fig.1

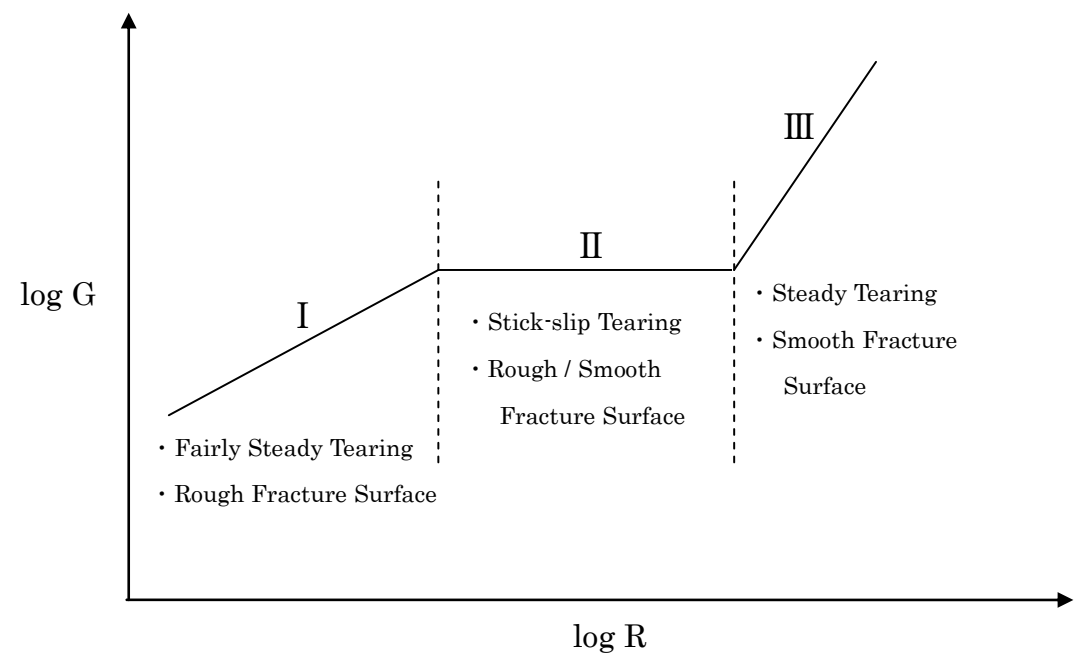

Fig. 2

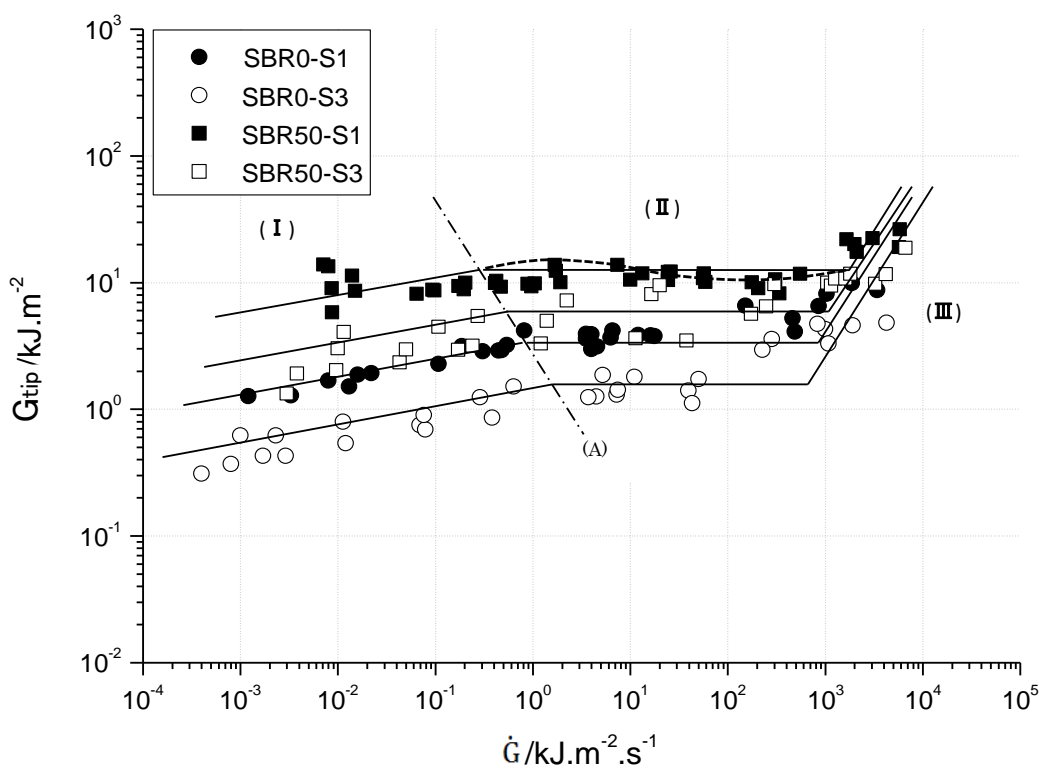


Fig. 3

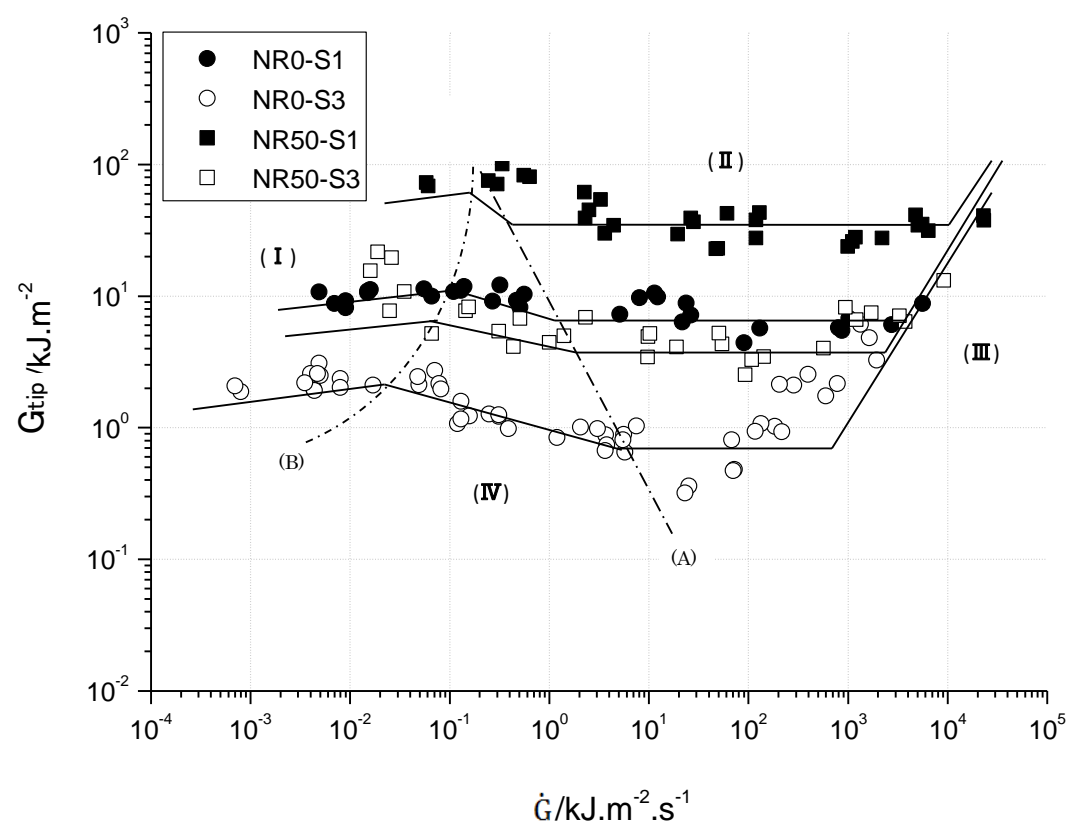

Fig.4a

\begin{tabular}{|c|c|c|c|}
\hline & Brittle Zone & Transition Zone & Ductile Zone \\
\hline & & & \\
\hline
\end{tabular}


Fig.4b

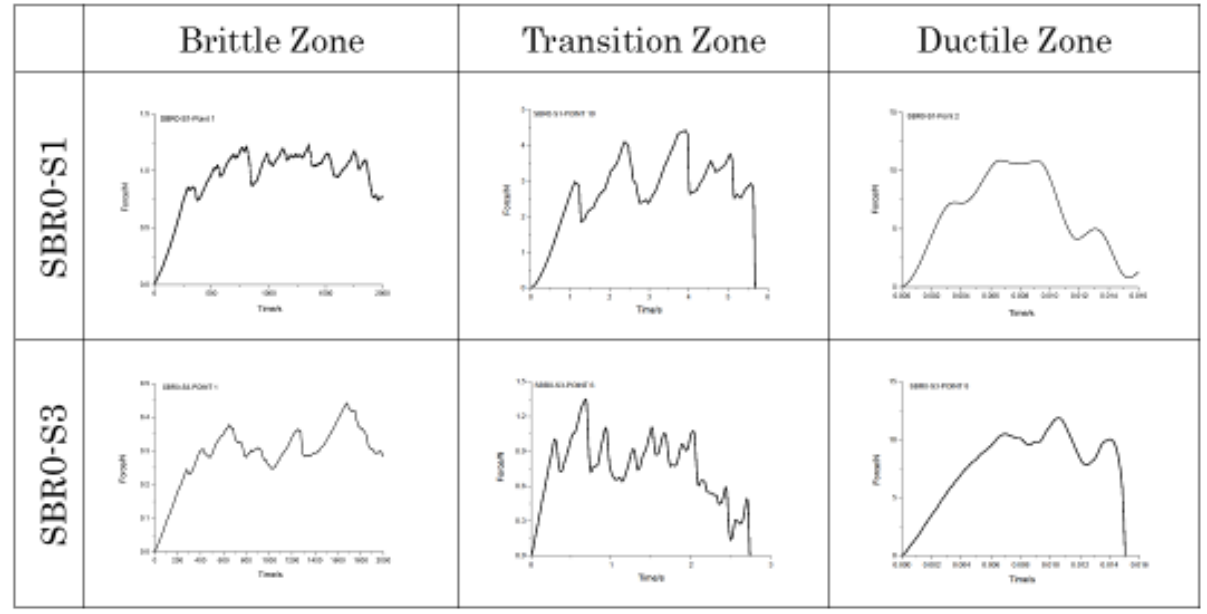

Fig.5

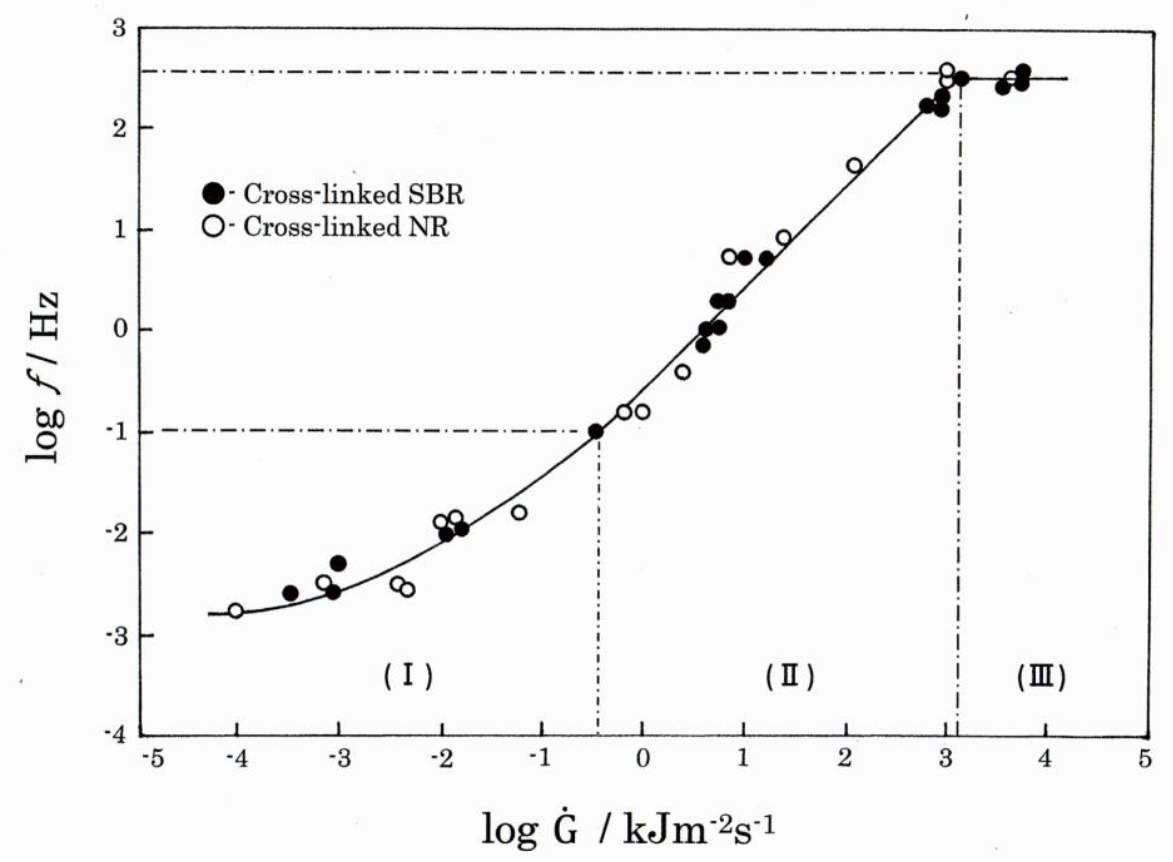


Fig.6

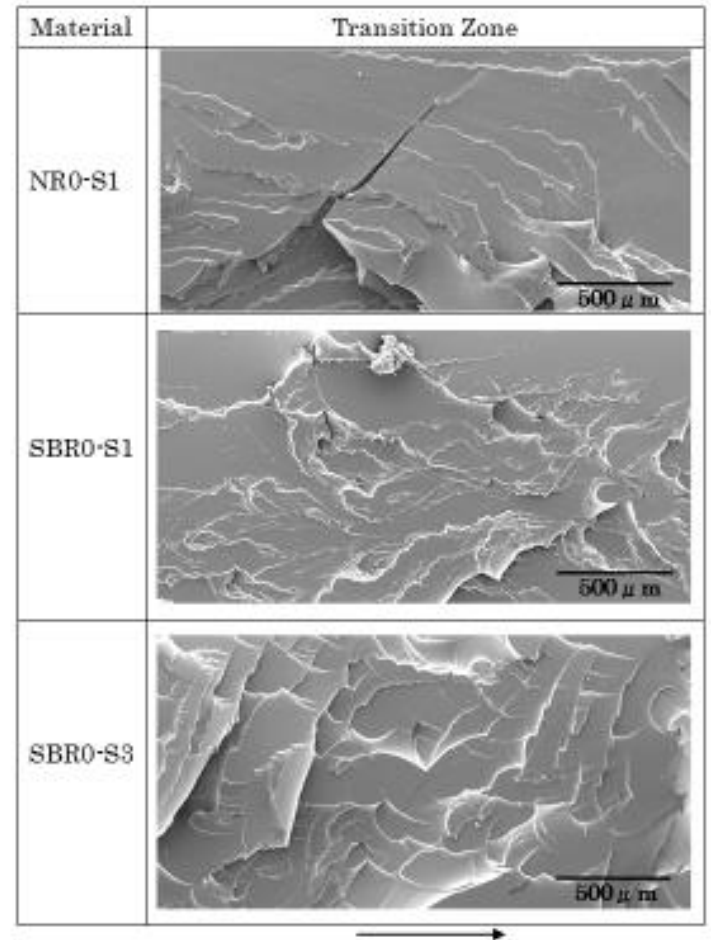

Fig.7

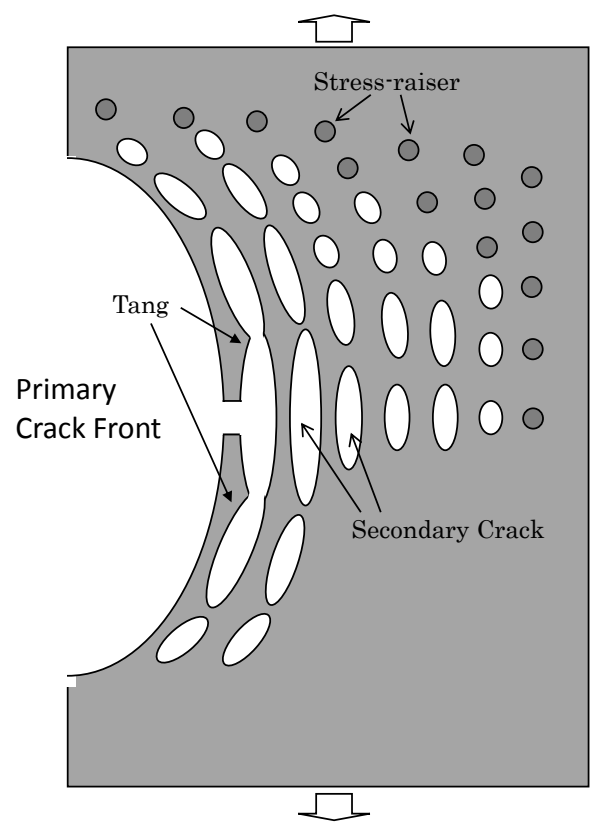


Fig.8

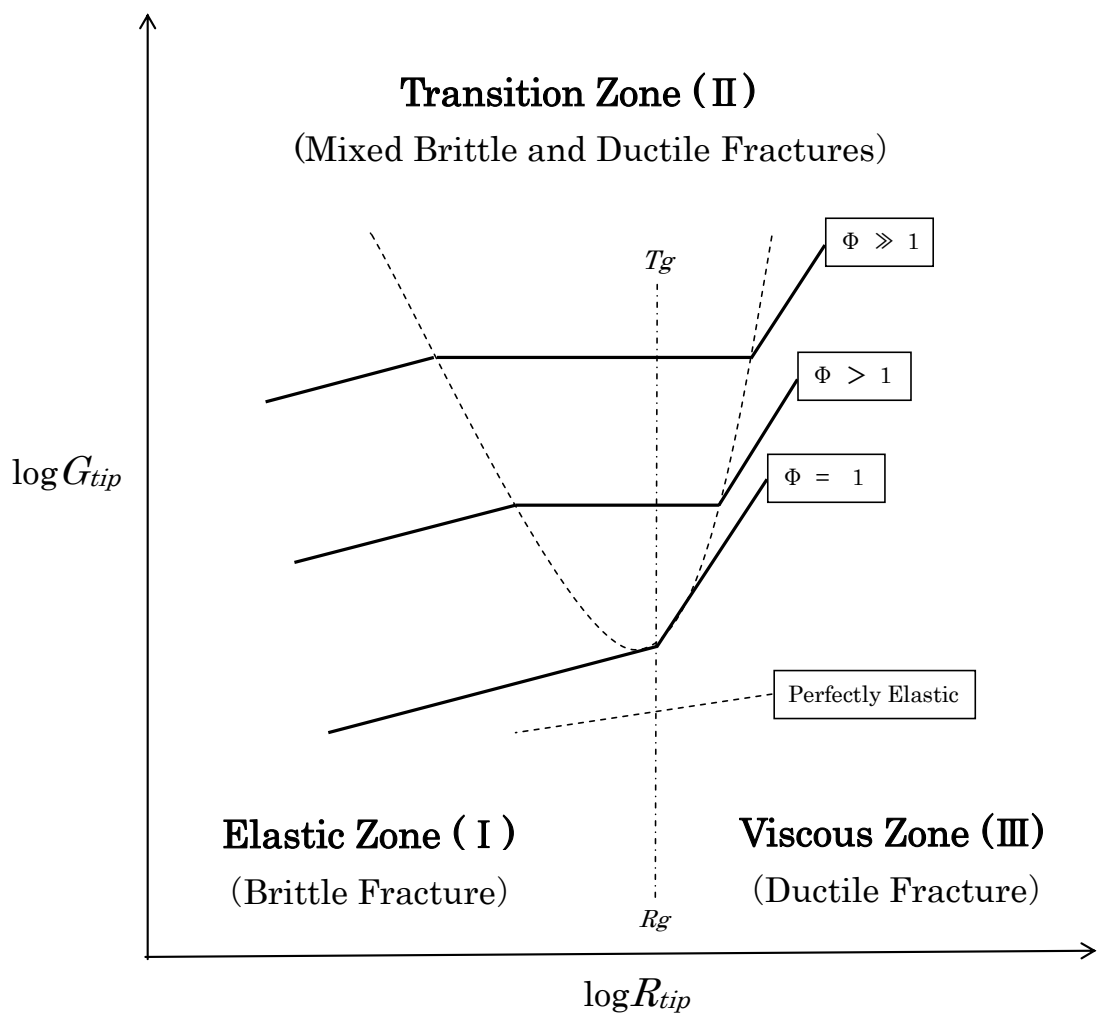

Fig.9

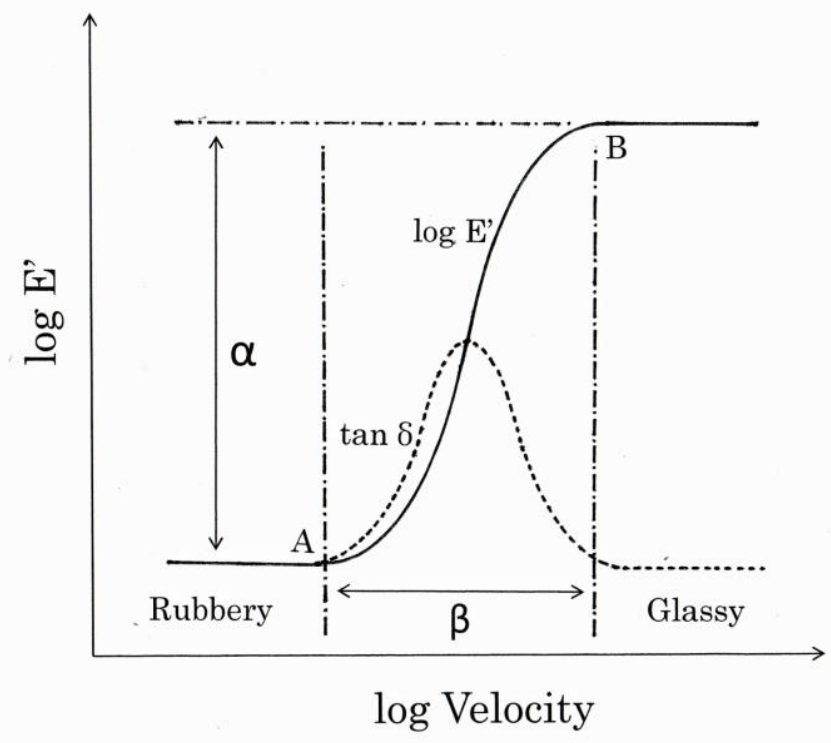


Fig.10

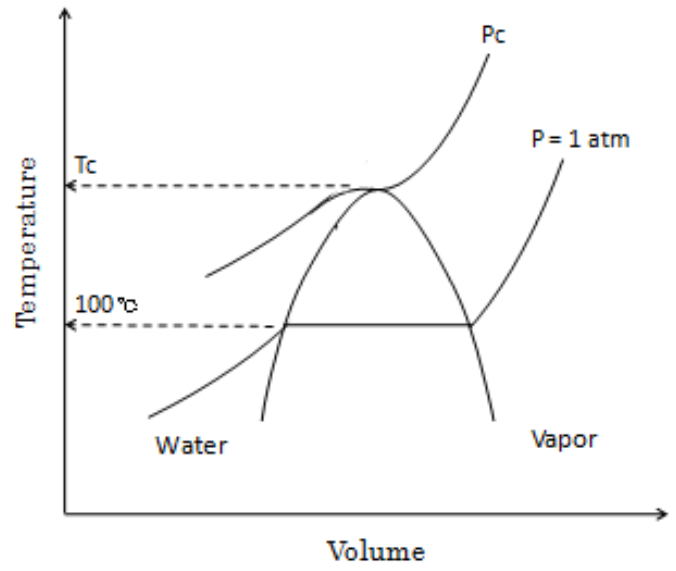

Fig.11
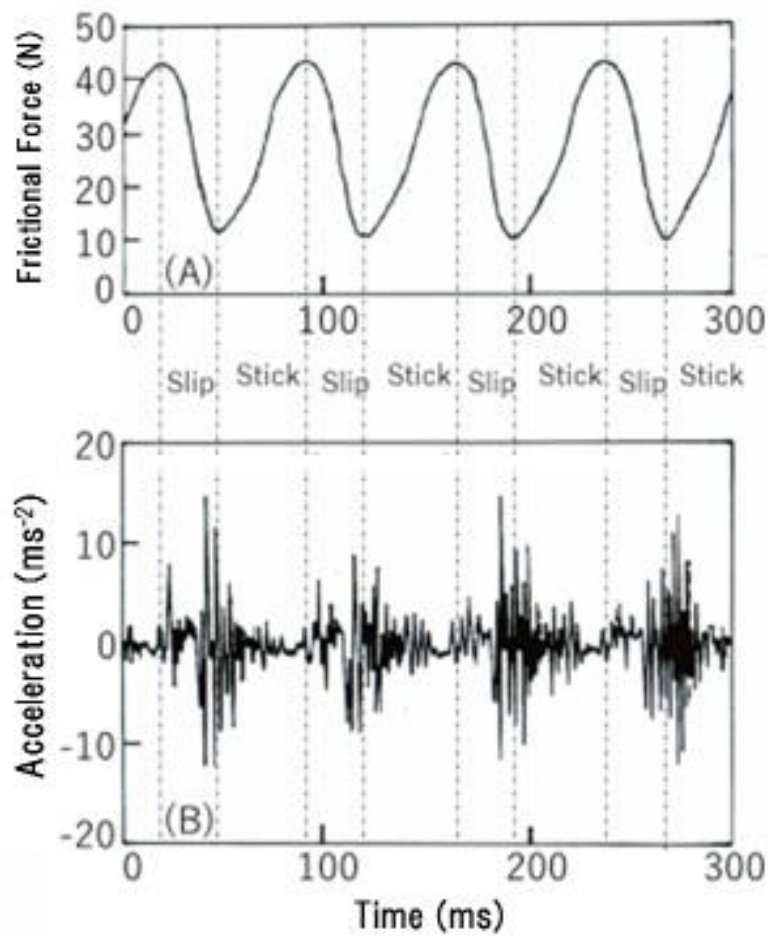